\title{
RELAÇÃO ENTRE NÍVEL DE ATIVIDADE FÍSICA E DESEMPENHO NO TESTE DE AVALIAÇÃO FÍSICA DE MILITARES
}

\section{The relationship between physical activity level and performance of millitaries in physical evaluation tests}

\section{Carlos Ricardo Gomes de Souza Rocha, Cíntia de La Rocha Freitas, Miguel Comerlato}

\section{RESUMO}

O presente estudo teve como objetivo verificar a relação entre o nível de atividade física (AF) e o desempenho no Teste de Avaliação Física (TAF) de militares do Exército Brasileiro. A amostra, composta por 121 militares, voluntários, integrantes de uma unidade militar da guarnição de Porto Alegre, caracterizou-se por indivíduos com idade média de 20,62 anos $( \pm 3,83)$, estatura média de $1,74 \mathrm{~m}( \pm 0,05)$, peso médio de $73,17 \mathrm{~kg}( \pm 9,7)$ e índice de massa corporal com média de $24,24 \mathrm{~kg} / \mathrm{m}^{2}( \pm 2,94)$. Para avaliação do nível de AF, foi utilizado o questionário internacional de atividades físicas em sua versão curta (IPAQ versão 6). Como determinante do desempenho físico, usou-se a média dos resultados individuais obtidos nos três TAF de 2007, compostos de teste de capacidade aeróbia (corrida de 12 minutos), teste de flexão de braços sobre o solo, teste de flexão abdominal e teste de flexão dos braços em barra fixa. A análise dos dados coletados evidenciou os seguintes resultados: houve uma predominância de indivíduos no peso normal $(66,09 \%)$ em relação aos classificados abaixo do peso $(0,87 \%)$, com sobrepeso $(26,96 \%)$ ou obesos $(6,09 \%)$. Já no tocante à prática de $A F$, houve uma predominância de indivíduos categorizados como muito ativos $(89,26 \%)$, em relação aos ativos $(9,09 \%)$ ou insuficientemente ativos $(1,65 \%)$. $\mathrm{Na}$ análise do desempenho físico individual, houve predominância de indivíduos com boa aptidão física (37,19\%), quando comparados àqueles com desempenho excelente $(21,49 \%)$, muito bom $(25,62 \%)$, regular $(4,13 \%)$ ou insuficiente (11,57\%). Quanto à análise da relação entre as variáveis propostas, houve apenas significância entre o nível de AF e o resultado do teste de corrida de 12 minutos $(r=0,203 ; p=0,026)$. Portanto, é possível concluir que não há relação significativa entre a prática de AF e o desempenho no teste de avaliação física do Exército Brasileiro.

Palavras-chave: Militares, Atividade Física, Avaliação Física.

\section{ABSTRACT}

This study aimed to examine the relationship between the level of physical activity (PA) and the performance test of Brazilian Army militaries in physical evaluation test (PET). The sample, made by 121 military volunteers members of a military unit in the garrison of Porto Alegre, is characterized by individuals with average age of 20.62 years $( \pm 3.83)$, average stature of $1.74 \mathrm{~m}( \pm 0.05)$, average weight of 73.17 kilograms $( \pm 9.7)$ and body mass index with an average of $24.24 \mathrm{~kg} / \mathrm{m}^{2}$ ( \pm 2.94$)$. To assess the level of PA, it was used the international physical activities questionnaire - short version (IPAQ version 6). As a determinant of physical performance, the average of individual results obtained in the three PETs of 2007 was used. It consisted of an aerobic capacity test (race, 12 minutes), a push-up test, a sit-up test and a pull-up test. The analysis of the data collected showed the following results: there was a predominance of people in the normal weight $(66.09 \%)$ over those classified under the weight $(0.87 \%)$, overweight $(26.96 \%)$ or obese $(6.09 \%)$. In regard to the practice of PA, a predominance of individuals categorized as very active $(89.26 \%)$, in relation to active $(9.09 \%)$ or insufficiently active (1.65\%). In the analysis of individual physical performance, there was a predominance of individuals with good physical fitness (37.19\%) when compared to those individuals with excellent performance $(21.49 \%)$, very good $(25.62 \%)$, regular $(4.13 \%)$ or insufficient $(11.57 \%)$. As for the analysis of the relationship between the proposed variables, there was only significance between the level of PA and the result of the running test of 12 minutes $(p=0.026)$. Therefore, it is possible to conclude that the practice of PA presents no significant relationship with the performance in the test of physical fitness of the Brazilian Army.

Key words: Military, Physical Activity, Physical Fitness.

Universidade Luterana do Brasil - Canoas - RS - Brasil.

Revista de Educação Física 2008 Set; 142:19-27. Rio de Janeiro (RJ) - Brasil.

Recebido em 20.03.2008. Aceito em 04.07.2008. 


\section{INTRODUÇÃO}

Sabe-se, hoje, que o condicionamento físico, em todos os Exércitos, é uma prioridade, visto que visa a saúde de seus militares, buscando capacitá-los para o exercício da profissão.

Ciente do número significativo de indivíduos, sendo grande parte composta por jovens que ingressam, anualmente, nas Forças Armadas, por força da lei em razão do serviço militar obrigatório, no Brasil, o Exército Brasileiro, no desempenho de seu papel como instituição engajada na formação social, vem se empenhando em preparar os militares, capacitando-os a contribuir no desenvolvimento do país e colaborando, sobremaneira, na construção de uma sociedade mais saudável.

A saúde pública tem sido tema de muitos comentários e especulações durante as últimas décadas. Conforme afirma Glaner (1), o trinômio "atividade física, aptidão física e saúde" tem sido objeto de inúmeros estudos, com diferentes delineamentos, em diversos países. Embora haja uma reconhecida complexidade conceitual de saúde, dificultando o entendimento das múltiplas facetas que a envolvem, parece haver um ponto pacífico quando o assunto envolve os benefícios de uma vida ativa.

A prática regular de exercícios está diretamente relacionada aos benefícios para a saúde. Já o sedentarismo e a inatividade física têm evidenciado uma forte correlação com os fatores de risco, tais como doenças coronarianas, entre outras alterações cardiovasculares ${ }^{(2)}$.

De acordo com Powers e Howley (3) , algumas investigações, realizadas nas décadas de 1980 e 1990, evidenciaram que indivíduos sedentários têm quase o dobro de chance de apresentar doença coronariana, se comparados aos fisicamente ativos. Além disso, eles apresentam risco relativo similar ao de tabagistas, de hipertensos e de indivíduos com altos níveis de colesterol sérico.

Por conseqüência da modernização dos meios de transporte e produção, houve uma redução da atividade física como meio de locomoção e no trabalho. Mesmo com a grande difusão e o conhecimento dos benefícios de um estilo de vida mais ativo, os indivíduos têm procurado cada vez menos a prática de atividades de lazer mais ativas, adotando um estilo de vida mais sedentário ${ }^{(4)}$.
Segundo Bloch, citado por Silva e Malina ${ }^{(5)}$, estudos, com amostras de diversas localidades brasileiras, indicam uma prevalência do sedentarismo em adultos, girando em torno de $70 \%$ durante o seu tempo de lazer. Diante disso, várias investigações vêm se desenvolvendo, objetivando analisar a aptidão física de militares e relacioná-la com os padrões de treinamento e indicadores de saúde ${ }^{(6,7)}$, visto que estudos longitudinais apontam uma relação direta e favorável entre o nível de aptidão física, o grau de atividade física praticada e a saúde ${ }^{(8)}$.

Entre os inúmeros fatores de risco relacionados com o sedentarismo, parece haver um que apresenta uma relação mais estreita que os demais: a obesidade. Este é o fator desencadeante de outros fatores secundários, tais como doenças cardiovasculares, diabetes melito tipo 2, distúrbios no aparelho locomotor, entre outros ${ }^{(9)}$.

Ainda, Meléndez, Pimenta e Kac ${ }^{(9)}$ apresentam a obesidade como um grave problema de saúde pública em países desenvolvidos, crescente em países em desenvolvimento, por decorrência da redução do nível de atividade física habitual e pela ingestão de alimentos de alto teor calórico. Além disso, descrevem um aumento na prevalência de sobrepeso em adultos brasileiros acima de 18 anos.

É justamente sobre este prisma que a prática de atividades físicas regulares atua, já que evidências epidemiológicas têm sugerido a existência de uma relação inversa entre o gasto energético e a gordura corporal, sendo esta melhor distribuída em indivíduos fisicamente ativos ${ }^{(3)}$.

Diante de tais evidências, torna-se coerente e necessário traçar investigações a fim de se compreender as relações entre a prática da atividade física, como instrumento promotor da saúde, e suas implicações sobre os fatores de risco atrelados ao sedentarismo. Só assim, tornar-se-á possível propor medidas eficientes e eficazes para implementar a saúde coletiva, contribuindo para o bem-estar comum.

No ambiente militar, a saúde e a aptidão física são fatores de suma importância. O treinamento físico, desenvolvido nas organizações militares (OM) brasileiras, por destinar-se à melhoria do desempenho da tropa no cumprimento de sua missão constitucional, possui um foco operacional mais evidente, o que dificulta a aplicação de princípios do treinamento físico como o da individualidade 
biológica. Contudo, como sua influência sobre a saúde atende, de melhor forma, aos interesses do militar, relacionando-se com o seu bem-estar e, conseqüentemente, melhorando a qualidade de vida de seus praticantes, tais benefícios tornam-se mais relevantes e duradouros ${ }^{(10)}$.

Diante disto, sabendo que conhecer o nível de aptidão física de seus militares é uma preocupação do Exército Brasileiro, as OM realizam, três vezes ao ano, o Teste de Avaliação Física (TAF).

Segundo Brasil ${ }^{(10)}$, o TAF tem por finalidade estabelecer os padrões de desempenho físico individual que orientarão o desenvolvimento do treinamento físico no Exército. Ou seja, servir de instrumento, através de uma verificação individual, para o acompanhamento do Treinamento Físico Militar (TFM) realizado pela tropa.

Em geral, existem dois métodos, tidos como principais, para a avaliação da aptidão e do desempenho físico. $O$ primeiro é a avaliação laboratorial das capacidades fisiológicas que, apesar de oferecer informações mais precisas e detalhadas, é de difícil aplicação e custo elevado. O segundo método são os testes de campo do condicionamento físico geral, os quais incluem várias mensurações que exigem demandas básicas de desempenho e que, além de serem de fácil aplicação em grupos numerosos, facilitam a interpretação dos dados por parte dos avaliadores ${ }^{(3)}$.

Alguns pesquisadores afirmam que a categorização do nível de atividade física por resultados, em testes de aptidão, traz limitações, visto que o desempenho é influenciado pelo estágio de maturação sexual, motivação, habilidade no teste, entre outros fatores ${ }^{(11)}$.

Entretanto, a avaliação não deve ser encarada simplesmente como um processo para atribuição de notas e conceitos. Ela deve ser entendida como um meio para observar o progresso, permitindo o entendimento e possibilitando intervenções no intuito de atingir determinados objetivos ${ }^{(12)}$.

No âmbito do Exército, o TAF é realizado conforme preconiza a Portaria ํo. 223, de 23 de dezembro de 2005, que entrou em vigor no ano de 2008.

Sendo realizado três vezes ao ano, o teste é aplicado em dois dias consecutivos: no primeiro dia, são realizadas as etapas de corrida de 12 minutos, flexão de braços e flexão abdominal; e, no segundo dia, a flexão na barra fixa e a Pista de Pentatlo Militar (13).

Segundo Rocha ${ }^{(14)}$, o teste de corrida, que consiste em correr ou andar a maior distância possível em 12 minutos, também conhecido como teste de Cooper, visa avaliar a resistência aeróbia do indivíduo.

Para o ambiente militar, esta valência física é de suma importância, uma vez que indivíduos melhor condicionados aerobiamente obtêm um melhor desempenho na realização de determinadas tarefas físicas mais complexas, que exigem um maior grau de atenção e concentração, quando comparados a indivíduos mal condicionados ${ }^{(15)}$.

Os testes de flexão de braços, de flexão na barra e de flexão abdominal destinam-se a verificar o nível de força e a resistência muscular localizada de determinados grupos musculares específicos, em diferentes situações ${ }^{(16)}$.

Ainda, Oliveira ${ }^{(16)}$ afirma que, no ambiente militar, a resistência muscular absoluta é uma valência bastante relevante, pois cargas típicas, como munições e armamentos, são carregadas por soldados, sendo seu peso sempre o mesmo, independente da força individual do soldado. Assim, soldados mais fortes terão uma maior capacidade para alta intensidade, qualidade requerida para que se levante e carregue estas cargas.

Percebe-se, então, que a aplicação de testes tendo por objetivo a avaliação individual e coletiva de grupos homogêneos e/ou heterogêneos, possui resultados bastante significativos, seja para nortear o planejamento do treinamento e identificar lideranças, ou seja do ponto de vista motivacional, já que os avaliados parecem demonstrar um alto nível de satisfação em executar e em saber seus resultados nos testes ${ }^{(12)}$.

Conhecer a capacidade física de seus homens, bem como o número de inaptos fisicamente a desempenhar tarefas mais árduas, é fundamental para o processo de tomada de decisão de um comandante. Portanto, a aptidão física de uma tropa deve ser avaliada sistematicamente ${ }^{(17)}$. Diante de tal constatação, sabendo-se da importância da prática de atividades físicas regulares e dos riscos provenientes de um estilo de vida sedentário, o presente estudo destina-se a observar o nível de atividade física (AF) de militares de uma unidade do Exército Brasileiro, da guarnição de Porto Alegre/RS (1 ${ }^{\text {a }}$ Companhia de Guardas), comparando com o desempenho obtido por estes militares no TAF, a fim de verificar se há alguma 
relação significativa entre ambos e identificar se os instrumentos utilizados são apropriados para esta população.

\section{METODOLOGIA}

\section{População e amostra}

A população desta pesquisa é caracterizada por militares da ativa do Exército Brasileiro, oriundos de uma unidade da guarnição de Porto Alegre, RS.

A amostra foi selecionada aleatoriamente, sendo composta por 121 militares da 1ㄹ Companhia de Guardas, os quais obtiveram o parecer médico favorável à realização dos Testes de Aptidão Física (TAF) do ano de 2007. Todos os indivíduos preencheram e assinaram o termo de consentimento livre e esclarecido (TCLE), tendo sido contemplados todos os procedimentos éticos para pesquisa em seres vivos, de acordo com a Resolução 196/96, Conselho Nacional de Saúde.

\section{Instrumentos e materiais}

Para a verificação do nível de atividade física, foi aplicado o Questionário Internacional de Atividade Física (IPAQ), em sua forma curta (versão 6), originalmente desenvolvido com a finalidade de estimar o nível de prática habitual de atividade física de populações de diferentes países $^{(18)}$.

O desempenho físico foi avaliado através do TAF do Exército Brasileiro, em conformidade ao prescrito na Portaria Ministerial número 739, do Estado-Maior do Exército, de 16 de setembro de 1997, em vigor na data da coleta.

O TAF é composto pelas seguintes avaliações: a) Teste de corrida de 12 minutos; b) Teste de flexão de braços; c) Teste de abdominal; d) Teste de flexão na barra. A categorização dos resultados foi feita levando-se em consideração os resultados obtidos nos três testes de 2007, com base nos índices das tabelas de avaliação física, preconizados pelo documento supracitado.

Além destes testes, é também previsto, no TAF, a execução de um teste em pista de pentatlo militar, não tendo, entretanto, o presente estudo, analisado a execução desta prova, em virtude das peculiaridades da OM em que foi realizada a pesquisa.

Para fins de ilustração, foram coletados, ainda, o peso corporal e a estatura, para o cálculo do índice de massa corporal (IMC), de maneira a traçar um perfil da amostra. Para isso, foi utilizada uma balança com precisão de 100 gramas (Filizola) e um estadiômetro com precisão em milímetros (Filizola). O IMC foi calculado com base na seguinte fórmula: peso $(\mathrm{kg})$ / estatura $(\mathrm{m})^{2}$. Sua categorização foi baseada nos índices padronizados pela Organização Mundial da Saúde ${ }^{(19)}$.

\section{Plano de coleta dos dados}

Inicialmente, foi estabelecido um contato com o comando da $1^{\text {a }}$ Companhia de Guardas, solicitando a autorização para a coleta de dados junto ao efetivo da OM. Tal contato teve por objetivo esclarecer o comando da unidade sobre os objetivos e a relevância do estudo em questão.

Concluídas as formalidades e após a aprovação deste estudo pelo Comitê de Ética em Pesquisa da ULBRA / Canoas (protocolo número 2007-338H), a coleta dos dados para a pesquisa seguiu o seguinte planejamento:

1. Preenchimento do TCLE;

2. Aplicação do IPAQ, através da técnica de auto-administração orientada, para todo o efetivo de militares da OM;

3. Coleta do peso corporal e da estatura para traçar o perfil do universo avaliado, classificando-os quanto ao IMC;

4. Avaliação médica para verificar a aptidão dos militares da OM para a realização das provas do TAF, excluindo da amostra da pesquisa, neste momento, os indivíduos com parecer inapto; e

5. Execução do TAF em dois dias consecutivos.

Os testes (TAF) foram aplicados nas seguintes condições:

1ㅇ dia $^{\circ}$

- Corrida de 12 minutos: na posição inicial de pé, cada militar correu ou andou a distância máxima, no tempo de 12 minutos, podendo interromper ou modificar o ritmo de corrida. A prova foi realizada em piso duro e plano, com as distâncias marcadas de 50 em 50 metros, sendo considerado, como resultado final, a próxima marca a ser ultrapassada pelo militar. Para marcação das distâncias, foi utilizada trena de 50 metros, anteriormente aferida. $\mathrm{O}$ uniforme para a realização da prova foi o previsto para a prática de 
treinamento físico no Exército e calçado adequado para corrida.

- Flexão de braços: em terreno plano, liso e na sombra, o militar se colocou em decúbito ventral, apoiando o tronco e as mãos no solo, ficando as mãos ao lado do tronco, com os dedos apontados para frente e os polegares tangenciando os ombros, permitindo, assim, que as mãos ficassem com um afastamento igual à largura do ombro. Após adotar a abertura padronizada dos braços, o indivíduo erguia o tronco, até que os braços ficassem estendidos, mantendo os pés unidos e apoiados sobre o solo. Para a execução, o militar abaixava o tronco e as pernas ao mesmo tempo, flexionando os braços, paralelamente ao corpo, até que o cotovelo ultrapassasse a linha das costas, ou o corpo encostasse o solo. Os braços eram, de novo, totalmente estendidos, erguendo simultaneamente o tronco e as pernas, quando, então, era completada uma repetição. Cada militar executou o número máximo de flexões de braços sucessivas, sem interrupção do movimento. O ritmo das flexões, sem paradas, foi opção do militar, não havendo limite de tempo. O uniforme utilizado foi o mesmo descrito para a corrida.

- Flexão abdominal: o indivíduo tomou a posição deitado, em decúbito dorsal, pernas e braços estendidos, braços acima da cabeça com o dorso das mãos tocando o solo. Para a execução, o avaliado realizou a flexão abdominal até que as a linha dos cotovelos ultrapassasse a linha dos joelhos flexionados, retornando à posição inicial, quando foi completada uma repetição. Cada militar executou as flexões abdominais, sem interrupção do movimento, em seu ritmo. O uniforme foi o mesmo descrito no item anterior.

- 2ำ Dia:

- Flexão na barra fixa: o indivíduo, sob a barra, a empunhou com pegada em pronação, com o polegar envolvendo a mesma. As mãos permaneceram com um afastamento, entre si, correspondente à largura dos ombros, sendo o corpo mantido estático. Após a ordem de iniciar, o militar executou uma flexão dos braços na barra, até que o queixo ultrapassasse completamente a barra (estando a cabeça na posição natural, sem hiperextensão do pescoço), descendo o tronco, imediatamente, até que os cotovelos ficassem completamente estendidos, quando, então, foi completada uma repetição. $O$ ritmo das flexões de braços na barra foi opção do militar e não houve limite de tempo. O uniforme foi o mesmo previsto nas atividades anteriores.

Após o término dos testes, foi feita a análise e a interpretação do IPAQ, com base nos critérios do próprio instrumento, dividindo os indivíduos em três grupos: insuficientemente ativos, ativos e muito ativos.

Em seguida, foram analisados os resultados obtidos nos três TAF do ano de 2007, sendo incluídos apenas os indivíduos que realizaram pelo menos dois dos três testes previstos. Para fins de análise, adotou-se, como critério, a média obtida em cada etapa dos três testes (no caso de resultados diferentes) ou a moda (no caso de repetição de um mesmo índice). Após este procedimento, realizou-se a conceituação em conformidade à legislação vigente para a instituição.

Finalizando, foi procedida uma comparação entre os resultados dos dois instrumentos de coleta, objetivando verificar a existência, ou não, de relação entre ambos.

\section{Tratamento estatístico}

Os dados do IPAQ e do TAF foram processados e submetidos à análise, através da estatística descritiva, com o objetivo de ordenar, sumarizar e descrever as informações coletadas e os resultados obtidos.

Como o estudo objetivava identificar uma possível relação entre o nível de atividade física habitual e o desempenho físico no TAF, foi realizado o teste de correlação linear de Pearson, através do pacote estatístico computacional SPSS (versão 10.0) para Windows, para verificar a relação entre o resultado do IPAQ e o resultado de cada etapa do teste (corrida, flexão de braços, flexão abdominal e flexão na barra). Já, para verificar a relação entre o resultado do IPAQ e a conceituação final do TAF, foi utilizado a correlação de Spearman. Considerou-se o nível de significância de 0,05.

\section{RESULTADOS}

Com o objetivo de verificar a possível relação entre o nível de atividade física (AF) e o desempenho no Teste de Avaliação Física (TAF) de militares, foi procedida uma coleta de idade, estatura, peso e índice de massa corporal (IMC) dos indivíduos participantes do estudo, a fim de se 
TABELA 1

PERFIL DA AMOSTRA QUANTO À IDADE E MEDIDAS ANTROPOMÉTRICAS.

\begin{tabular}{lcccc}
\hline Variável & Média & Mínimo & Máximo & Desvio padrão \\
\hline Faixa etária (anos) & 20,62 & 18 & 43 & 3,83 \\
Estatura $(\mathrm{m})$ & 1,74 & 1,6 & 1,87 & 0,05 \\
Peso $(\mathrm{kg})$ & 73,17 & 51,2 & 107,4 & 9,7 \\
$\mathrm{IMC}\left(\mathrm{kg} / \mathrm{m}^{2}\right)$ & 24,24 & 18,1 & 33,8 & 2,94 \\
\hline
\end{tabular}

obter um perfil do universo. Após análise destes dados, foi possível verificar, conforme ilustrado na TABELA 1, que a amostra possuía uma faixa etária com média de 20,62 anos $( \pm 3,83)$, estatura média de 1,74 metros $( \pm 0,05)$ e peso corporal médio de 73,17 quilogramas $( \pm 9,7)$. Diante dos resultados de estatura e peso dos indivíduos, calculou-se o IMC dos mesmos, onde se obteve uma média de 24,24 $\mathrm{kg} / \mathrm{m}^{2}( \pm 2,94)$.

Com base nos resultados de IMC, a amostra foi distribuída em conformidade à classificação da Organização Mundial da Saúde (OMS). Conforme demonstra a TABELA 2, houve uma predominância de indivíduos no peso normal $(66,09 \%)$, seguido de um número significativo de indivíduos em situação de sobrepeso (26,96\%). Apenas uma minoria do universo avaliado foi classificada em condição de obesidade (6,09\%) e abaixo do peso $(0,87 \%)$.

Após o preenchimento do Questionário Internacional de Atividade Física (IPAQ), a amostra foi distribuída com base nos critérios do próprio instrumento. Nesta análise, conforme ilustrado na TABELA 3, a grande maioria dos indivíduos foi classificada como muito ativo $(89,26 \%)$, ficando apenas uma minoria classificada como ativo $(9,09 \%)$ ou insuficientemente ativo $(1,65 \%)$.
Baseado nos resultados dos três TAF do ano de 2007, traçou-se um perfil do universo avaliado, estabelecendo a média obtida em cada etapa do referido teste. De acordo com a TABELA 4, é possível identificar as seguintes médias: 3057,11 metros $( \pm 220,84)$ no teste de corrida de 12 minutos; 35,88 repetições $( \pm 5,52)$ no teste de flexão de braços; 63,17 repetições $( \pm 8,98)$ no teste de flexão abdominal; e 8,91 repetições $( \pm 2,93)$ no teste de flexão na barra fixa.

Depois de terem sido analisados os resultados individuais nos três TAF, a amostra foi classificada em relação ao nível de aptidão física, de acordo com os critérios preconizados pelo instrumento. Assim, a TABELA 5 mostra que houve uma prevalência de indivíduos categorizados no desempenho bom $(37,19 \%)$, seguido dos indivíduos com desempenho muito bom (25,62\%) e excelente $(21,49 \%)$. Houve, ainda, indivíduos com desempenho regular $(4,13 \%)$ e insuficiente $(11,57 \%)$.

Finalizando as análises, foi estabelecida correlações entre as variáveis apuradas, no intuito de verificar a influência entre elas. Conforme ilustra a TABELA 6 , houve uma relação positiva e significativa $(p=0,026)$, apesar de fraca $(r=0,203)$, apenas entre o nível de atividade física

TABELA 2

DISTRIBUIÇÃO DA AMOSTRA DE ACORDO COM O IMC.

\begin{tabular}{lcc}
\hline Classificação & $\mathbf{N}^{\circ}$ & $\%$ \\
\hline Abaixo do Peso & 1 & 0,87 \\
Peso Normal & 76 & 66,09 \\
Sobrepeso & 31 & 26,96 \\
Obesidade & 7 & 6,09 \\
Obesidade Grave & 0 & 0 \\
TOTAL & 115 & 100 \\
\hline
\end{tabular}




\section{TABELA 3}

DISTRIBUIÇÃO DA AMOSTRA DE ACORDO COM A ANÁLISE DO IPAQ.

\begin{tabular}{lcc}
\hline Classificação & $\mathbf{N}^{\circ}$ & $\%$ \\
\hline Insuficientemente Ativo & 2 & 1,65 \\
Ativo & 11 & 9,09 \\
Muito Ativo & 108 & 89,26 \\
TOTAL & 121 & 100 \\
\hline
\end{tabular}

TABELA 4

PERFIL DA AMOSTRA QUANTO AOS RESULTADOS OBTIDOS NOS TAF.

\begin{tabular}{lcccc}
\hline Variável & Média & Mínimo & Máximo & Desvio padrão \\
\hline Corrida $(m)$ & 3057,11 & 2520 & 3630 & 220,84 \\
Flexão de Braços & 35,88 & 19 & 60 & 5,52 \\
Flexão Abdominal & 63,17 & 27 & 92 & 8,98 \\
Flexão na Barra Fixa & 8,91 & 2 & 19 & 2,93 \\
\hline
\end{tabular}

TABELA 5

DISTRIBUIÇÃO DA AMOSTRA EM RELAÇÃO AO NÍVEL DE APTIDÃO FíSICA.

\begin{tabular}{lcc}
\hline Classificação & $\mathbf{N}^{\circ}$ & $\%$ \\
\hline Insuficiente & 14 & 11,57 \\
Regular & 5 & 4,13 \\
Bom & 45 & 37,19 \\
Muito Bom & 31 & 25,62 \\
Excelente & 26 & 21,49 \\
TOTAL & 121 & 100 \\
\hline
\end{tabular}

TABELA 6

CORRELAÇÕES ENTRE AS VARIÁVEIS ANALISADAS.

\begin{tabular}{lcc}
\hline Classificação & $\mathbf{r}$ & $\mathbf{p}$ \\
\hline IPAQ x IMC & -.087 & .354 \\
IPAQ x Corrida & .203 & $.026^{*}$ \\
IPAQ x Flexão de Braço & .124 & .176 \\
IPAQ x Flexão Abdominal & .099 & .282 \\
IPAQ x Flexão na Barra Fixa & .070 & .452 \\
IPAQ x Nível de Aptidão Física & .020 & .828 \\
\hline
\end{tabular}


aferido pelo IPAQ e o resultado obtido no teste de corrida de 12 minutos.

\section{DISCUSSÃO}

Diante do exposto, observa-se que há uma predominância de indivíduos classificados como muito ativos (TABELA 3), segundo os critérios do IPAQ. Tal resultado já era esperado, visto que o referido instrumento tem sua aplicação mais voltada para populações sedentárias, sendo pouco utilizado em populações mais específicas, o que compromete sua aplicabilidade na amostra em questão. Apesar de não ter sido encontrado nenhum estudo na literatura realizado com militares que evidenciasse isso, esperava-se que os mesmos obtivessem resultados acima da população comum.

Paralelo a isto, percebe-se, também, que a maioria dos indivíduos obteve uma classificação positiva na avaliação do TAF (TABELA 5). A literatura aponta inúmeras variáveis envolvidas no desempenho individual em testes de aptidão física. Fatores genéticos, estágio de maturação sexual, motivação, além de habilidade no teste, são fatores que podem interferir nestes resultados ${ }^{(11)}$.

Outro dado interessante, encontrado no presente estudo, foi o resultado da análise do IMC dos indivíduos. Percebe-se que houve uma predominância de indivíduos categorizados dentro do peso normal (TABELA 2). Entretanto, não houve uma correlação significativa entre o nível de AF e o IMC da amostra (TABELA 6).

Ao correlacionar o nível de AF dos indivíduos com os diferentes testes que compõem o TAF, não foi encontrada influência significativa entre eles, exceto para o teste de corrida de 12 minutos. Para esta atividade, apesar da baixa correlação, parece haver um nível de significância positivo
(TABELA 6). Ou seja, isto pode indicar que quanto mais ativo um indivíduo em suas atividades habituais, melhor pode ser o seu desempenho em testes de capacidade aeróbia, mais especificamente, no teste de 12 minutos de Cooper.

Morrow e Freedson, por exemplo, citados por Silva e Malina $^{(5)}$, descreveram que são baixos os coeficientes de correlação encontrados entre a aptidão cardiorrespiratória, medida pelo teste de 12 minutos de Cooper, e os instrumentos de medição da atividade física.

Outro fator que também explicaria a baixa relação para as outras etapas do TAF é a possível ineficácia do IPAQ em quantificar a atividade física habitual deste tipo de população, havendo necessidade de adaptá-lo ou buscar outros instrumentos mais adequados.

\section{CONCLUSÃO}

Com base no objetivo traçado para este estudo e na análise dos resultados obtidos, é possível concluir que não há relação entre a prática de atividades físicas, quantificada pelo IPAQ, e o desempenho de militares nos testes de avaliação física aplicados pelo Exército Brasileiro, possivelmente em razão da ineficácia do referido instrumento em quantificar o nível de AF desta população.

Contudo, parece haver uma correlação, ainda que fraca, entre o nível de atividade física com o resultado no teste de corrida de 12 minutos de Cooper, o que leva a crer que indivíduos com hábitos de vida mais ativos possuem uma melhor aptidão cardiorrespiratória, em comparação aos indivíduos menos ativos. Recomenda-se, portanto, que mais estudos, com outros instrumentos e delineamentos, sejam realizados com a intenção de esclarecer e aprofundar estas evidências.

\section{REFERÊNCIAS BIBLIOGRÁFICAS}

1. Glaner MF. Nível de atividade física e aptidão física relacionada à saúde em rapazes rurais e urbanos. Revista Paulista de Educação Física 2002; 16:76-85.

2. Assunção S, Cordas T, Araújo L. Atividade física e transtornos alimentares. Revista de Psiquiatria Clínica 2002; 29(1):4-13.

3. Powers SK, Howley ET. Fisiologia do exercício: teoria e aplicação ao condicionamento e ao desempenho. Tradução de Navarro F, Bacurau RFP. 3ª ed. São Paulo: Manole; 2000.

4. Oliveira ESA. Atividade física habitual e outros comportamentos relacionados à saúde dos servidores da Universidade Federal de Santa Catarina: tendência secular 1994-2004 [dissertação]. Florianópolis, SC: Programa de Pós-Graduação em Educação Física da Universidade Federal de Santa Catarina; 2005. 
5. Silva R, Malina R. Nível de atividade física em adolescentes do município de Niterói, Rio de Janeiro, Brasil. Cadernos de Saúde Pública 2000; 16(4).

6. Vieira G, Duarte D, Silva R. Efeitos de oito semanas de treinamento físico militar sobre o desempenho físico, variáveis cardiovasculares e somatório de dobras cutâneas de militares de força de paz do Exército Brasileiro. Revista de Educação Física do Exército 2006; 134: 31-2.

7. Rocha CRGS. Aptidão física relacionada à saúde em militares da 1aㅡ Companhia de Guardas [monografia]. Canoas, RS: Universidade Luterana do Brasil; 2005.

8. Bezerra Filho MJA. Níveis de aptidão física relacionados à saúde dos policiais militares que trabalham no serviço de rádio-patrulha do $5^{\circ}$ Batalhão de João Pessoa [monografia]. João Pessoa, Paraíba: Curso de especialização em segurança pública da Academia de Polícia Militar da Paraíba; 2004.

9. Meléndez GV, Pimenta AM, Kac G. Epidemiologia do sobrepeso e da obesidade e seus fatores determinantes em Belo Horizonte (MG), Brasil: estudo transversal de base populacional. Revista Panamericana de Salud Publica 2004; 16(5): 308-14.

10. Brasil. Portaria ํㅡ 223, de 23 de dezembro de 2005. Aprova a diretriz para o Treinamento Físico Militar do Exército e a sua avaliação. Brasília, DF: EGGCF - Boletim do Exército; 2006.

11. Fox K, Biddle S. The use of fitness tests. Educational and psychological considerations. Journal of Physical Education, Recreation and Dance 1988; 59(2): 46-53.

12. Shigunov V. Reflexões sobre os testes físicos em alunos universitários. Departamento de Educação Física, Centro de Desportos, Universidade Federal de Santa Catarina. Disponível em: URL: http://www.kinein.ufsc.br/edit01/artigo7.pdf. (19 jun. 2007).

13. Brasil. Portaria no. 739, de 16 de setembro de 1997. Aprova a diretriz para o Treinamento Físico Militar do Exército e a sua avaliação. Brasília, DF: EGGCF - Boletim do Exército; 1997.

14. Rocha PEC. Medidas e avaliação em ciências do esporte. 4aㅡ ed. Rio de Janeiro: Sprint; 2000.

15. Dias AC, Dantas EHM, Moreira SB, Silva VF. A relação entre o nível de condicionamento aeróbico, execução de uma pista de obstáculos e o rendimento em um teste de tiro. Revista Brasileira de Medicina do Esporte 2005; 11(6): 341-6.

16. Oliveira EAM. Validade do teste de aptidão física do Exército Brasileiro como instrumento para determinação das valências necessárias ao militar. Revista de Educação Física do Exército 2005; 131: 30-7.

17. Costa GMM, Machado AAB, Annes DB, Pereira MMM, Neto OP, Carvalho F, et al. Performance de militares do Exército Brasileiro no army physical fitness test. Revista de Educação Física do Exército 2004; 128: 60-4.

18. Guedes DP, Lopes CC, Guedes JERP. Reprodutibilidade e validade do Questionário Internacional de Atividade Física em adolescentes. Revista Brasileira de Medicina do Esporte 2005; 11(2): 151-8.

19. Organização Mundial de Saúde. Obesity: controlling the global epidemic. Disponível em: URL: http://www.who.int/nut/ obs.htm.

\section{Endereço para correspondência:}

Carlos Ricardo Gomes de Souza Rocha Rua da Gávea, 541

Porto Alegre - RS - Brasil

CEP 91760-040

e-mail: tensouza@yahoo.com.br cintiadelarocha@ig.com.br mcomerlato@terra.com.br 